\title{
Bullous Pemphigoid, Mucous Membrane Pemphigoid and Pemphigus Vulgaris: An Update on Pathobiology
}

\author{
Lauren Okon • Victoria Werth
}

Published online: 1 June 2014

(C) Springer International Publishing AG (outside the USA) 2014

\begin{abstract}
Pemphigoid and pemphigus vulgaris (PV) are autoimmune diseases that cause potentially debilitating erosions and blistering of the skin and/or mucous membranes. In bullous pemphigoid (BP), autoantibodies target components of the basement membrane zone (BMZ), most importantly the hemidesmosomal proteins, BP180 and BP230. Research efforts have uncovered some of the complex mechanisms that cause this disease, including the interplay between the humoral and cell-mediated immune responses, as well as the role of complement activation, inflammatory cell activation, and proteolytic enzymes. In mucous membrane pemphigoid, which preferentially targets mucous membranes, several antigenic targets along the BMZ have been identified. In PV, desmoglein autoantibodies play the most critical role in the disease, with various other target antigens acting adjunctively to exacerbate the disease by apoptotic signaling. The relationship between apoptosis and acanthosis has yet to be clearly defined, but they may promote each other to enhance disease
\end{abstract}

L. Okon $\cdot$ V. Werth

Philadelphia Veterans Affairs Medical Center, Philadelphia, PA, USA

L. Okon · V. Werth

Department of Dermatology, Perelman School of Medicine at the University of Pennsylvania, Philadelphia, PA, USA

\section{Werth $(\bowtie)$}

Department of Dermatology, Perelman Center for Advanced Medicine, Suite 1-330A, 3400 Civic Center Boulevard, Philadelphia, PA 19104, USA

e-mail: werth@mail.med.upenn.edu

\section{Okon $(\bowtie)$}

Department of Dermatology, University of Pennsylvania, 14 Penn Tower Room 1430, 1 Convention Ave, Philadelphia, PA 19104, USA e-mail: Lauren.okon@uphs.upenn.edu activity. This article provides an update on the pathogenesis of these diseases.

Keywords Pemphigoid · Pemphigus vulgaris · Pathobiology $\cdot$ Bullous pemphigoid

\section{PEMPHIGOID}

Introduction

Pemphigoid is a group of autoimmune diseases characterized by autoantibodies that target specific structural components of the dermal-epidermal junction. This complex interaction leads to separation of the dermis from the epidermis, which manifests clinically as tense blisters and erosions. This discussion will focus on two of the pemphigoid diseases: bullous pemphigoid (BP) and mucous membrane pemphigoid (MMP).

Bullous Pemphigoid (BP)

\section{Target Autoantigens}

$\mathrm{BP}$, the most common autoimmune blistering disease, is caused by autoantibodies against components of the skin basement membrane zone (BMZ) [1]. Clinically, this disease is characterized by tense blisters and erosions, erythema, and pruritic urticarial plaques occurring on the whole body. In contrast to pemphigus vulgaris (PV), BP does not exhibit the Nikolsky sign, meaning that friction applied to non-lesional skin does not lead to intraepidermal extension of blisters.

The hemidesmosomal proteins, BP180 and BP230, have been identified as the target antigens in BP [1, 2]. Hemidesmosomes are membrane-associated, rivet-like 
structures located on the inner basal surface of keratinocytes that link the cell to the extracellular matrix. Chromosomal mapping studies have localized the BP180 gene to the long arm of chromosome 10, locus 10q24.3, and the BP230 gene to the short arm of chromosome 6 , locus 6p11-12 [3, 4]. These genes have been shown to be unrelated through epidermal complementary DNA (cDNA) cloning studies [5, 6]. BP180 is a 180-kDa type II transmembrane protein that spans the lamina lucida and then extends into the lamina densa of the BMZ. This hemidesmosomal antigen of type XVII collagen (also known as COL17) consists of a globular intracytoplasmic domain and an extracellular component with 15 collagenous domains [7]. In epitope mapping studies, the non-collagenous 16A (NC16A) extracellular region of BP180 was found to be the major pathogenic epitope in BP [8]. Memory B cells specific to this domain have been identified in BP patients [9]. The titer of anti-BP180 NC16A antibodies has repeatedly been shown to correlate with BP disease severity $[10,11]$. Additional antibodies outside the NC16 domain have also been detected in the sera of most BP patients [12]. IgG reactivity with the C-terminal portions of BP180 appears to be linked to mucosal involvement of the disease. Conversely, reactivity with the intracellular domain is seen in early clinical presentation of the disease [13]. Most patients' sera also contain IgA and IgE antibodies to BP180 [14-16]. BP patients can also show reactivity to BP230. BP230 is a $230 \mathrm{kDa}$ intracellular plakin protein that comprises part of the hemidesmosomal plaque. Epitopes are preferentially localized to the C-terminal domain, with both $\operatorname{IgG}$ and $\mathrm{IgE}$ reactivity detected [17]. Epitope spreading can explain the presence of several antigenic sites on both $\mathrm{BP} 180$ and $\mathrm{BP} 230$, as the disease persists [18].

\section{Humoral Immune Response}

There is extensive evidence to support the pathogenic role of BP180. The positive correlation between the titer of $\operatorname{IgG}$ (predominately IgG1 and IgG4) to extracellular epitopes of BP180 and BP disease severity is well recognized. In vitro studies and animal models have demonstrated the critical role of Fc receptor-mediated effects in blister formation [19-21]. Liu et al. described an animal model in which purified rabbit anti-murine BP180 was passively transferred into neonatal mice. The injected mice subsequently developed clinical findings akin to human BP [22]. This model has highlighted the pivotal role of the following in the formation of blisters: complement activation at the dermal-epidermal junction, neutrophils, macrophages, and various proteases, including neutrophil elastase (NE), matrix metalloproteinase 9, plasmin, $\alpha 1$-proteinase inhibitor, and mast cell protease- 4 [21, 23]. Two mouse models and a model in human keratinocytes have supported a pathogenic role of anti-BP180 IgE in BP [24-26]. Some evidence showing the positive clinical effects of omalizumab, a humanized monoclonal antibody (mAb) to the IgE high-affinity receptor, also supports the association of IgE [27].

In contrast to BP180, the pathogenic role of BP230 is less well understood. BP230 has been shown to cause an inflammatory response in rabbit models only following prior epidermal injury. These passive transfer models have failed to induce BP-like clinical changes, as has been shown with antiBP180 [28]. BP230 is an intracytoplasmic antigen, and thus it has been postulated that an attack on the extracellular BP180 domain is critical as a preceding event to trigger BP230 autoantibody formation. Once this event has occurred, antiBP230 can theoretically induce a local inflammatory response, even in the absence of anti-BP180 [29]. Conversely, Kiss et al. reported subepidermal blistering in neonatal mice injected with rabbit anti-BP230, suggesting that these antibodies may have access to their intracellular target antigen [30]. Clearly, there is a need for future studies to better elucidate the role of the autoantibodies in BP pathogenesis.

\section{Cell-Mediated Immunity}

The initiating trigger for the autoimmune response in BP is largely unknown. As with other autoimmune diseases, it is likely due to a breakdown of T- and B-cell tolerance to BP antigens, leading to autoantibody production and blister induction [29]. A deficit in the T-regulatory cells (Tregs) has been suggested as an inciting factor; however, research by Rensing-Ehl et al. showed no reduction in the number or function of Tregs in BP patients [31]. Other investigators have considered the role of toll-like receptors (TLRs), similar to their pathogenic role in pemphigus. In this model, autoreactive B cells are activated by B-cell receptor and TLR ligation by an endogenous TLR ligand. It is hypothesized that this leads to a breakdown of self-tolerance, activates autoreactive $\mathrm{T}$ cells, and initiates full-blown autoimmunity [32]. Autoreactive T cells that recognize the BP180 NC16A domain and show a T helper (Th)-1/Th2 mixed cytokine profile are recognized in both patients with BP as well as healthy controls. Th1 cytokines induce the secretion of IgG1 and IgG2, while Th2 cytokines control the secretion of IgG4 and IgE, and therefore the presence of anti-BP180 and anti-BP230 with IgG1, IgG4, and IgE isotypes supports the involvement of both Th1 and Th2 responses [29]. Elevated serum levels of many of these Th1 and Th2 cytokines have been correlated with BP disease severity [33]. A recent study by Li et al. found that BP patients had higher serum levels of follicular Th cells (Tfh) and interleukin (IL)-21 as compared with healthy controls. The authors also found that CD4+ T cells depleted of Tfh and anti-IL-21 neutralization antibody blocked T-cell-induced B-cell activation and secretion of BP antibodies in vitro [34•].

Th17 cells, which release cytokines such as IL-17, have also been implicated in playing an adjunctive role in the 
development of BP, accounting for neutrophil infiltration and eosinophil recruitment, which are characteristic of the disease. There is mounting evidence that IL-17 is involved in the initiation and perpetuation of several autoimmune diseases, including rheumatoid arthritis, systemic lupus erythematosus, psoriasis, and atopic asthma. IL-17 is responsible for the production of pro-inflammatory cytokines (tumor necrosis factor [TNF]- $\alpha$, IL-1, IL-6, IL-8, granulocyte-macrophage colony-stimulating factor), matrix metalloproteinases (MMP9, MMP-13), as well as the induction of Th2 cell activation [35]. Arakawa et al. found a greater degree of Th17 cellrelated inflammation and less Treg-related regulation in BP as compared with pemphigus [36]. A recent study by Zebrowska et al. observed a higher serum level of IL-17 in BP patients as compared with healthy controls, and also identified high levels of IL-17 expression in the epidermis of BP lesional and perilesional skin biopsies, but no IL-17 in the biopsies of healthy controls [37•]. Despite these findings, lesional Treg studies have yielded conflicting results, and future studies are needed to further elucidate the role of Th17.

Antibody production depends on the cooperation of $\mathrm{B}$ cells with CD4+ T cells. BP180 and BP230 antigens are detected by antigen-presenting cells, bound to major histocompatibility complex (MHC) class II and displayed on the surface of the cell. T cells recognize these epitopes, which trigger cytokine release and subsequently autoantibody production by B cells. Due to their role in the antigen-presenting process, human leukocyte antigen (HLA) genes likely represent a significant predisposing factor in $\mathrm{BP}$, and several studies have linked the HLA-DQ $\beta 1 * 0301$ allele to pemphigoid variants [38].

\section{Steps to Tissue Injury and Blister Formation in BP}

BP autoantibodies are believed to be pathogenic by the following four mechanisms: (1) complement activation; (2) inflammatory cell recruitment; (3) release of proteolytic enzymes; and (4) direct interference with autoantigen adhesion function.

\section{Complement Activation}

Past research has shown that BP autoantibodies fix complement in vitro and that direct immunofluorescence of perilesional skin shows $\mathrm{C} 3$ at the BMZ in nearly all $\mathrm{BP}$ cases $[39,40]$. Elements of both the classical and alternative complement pathways (including C1q, C4, C5, C5-9, factor B, $\mathrm{B} 1 \mathrm{H}$ globulin, and properdin) have been found in BP lesions [41]. Animal models demonstrated that rabbit anti-murine BP180 IgG was unable to induce lesions in C5-deficient mice, and that C5a-reconstituted mice became susceptible to autoantibody lesion induction [42].

\section{Inflammatory Cell Action}

Mast-cell degranulation has repeatedly been detected in BP lesional sites of both BP patients and animal models injected with anti-mBP180 antibodies [43, 44]. Studies have supported the notion that IgE anti-BP180 autoantibodies stimulate mastcell degranulation [45]. Antigen-specific histamine release has been observed only in patients with detectable serum $\operatorname{IgE}$ directed against the NC16A domain of BP180, which is known to be the target antigen in BP. In support of the role of mast cells, IgE-bearing mast cells and eosinophils have been detected in the dermis of BP patients [46]. Research has also shown that C3a and $\mathrm{C} 5 \mathrm{a}$ fragments can induce degranulation of mast cells, and, alternatively, that a deficiency in C5 prevents it. Mast cell degranulation leads to the subsequent release of a variety of inflammatory mediators, such as leukotrienes, platelet-activating factor, TNF $\alpha$, and other cytokines, which in turn recruit neutrophils and eosinophils to the site of lesion formation [29].

Neutrophils have been shown to play a critical role in dermal-epidermal separation in an in vitro cryosection model of BP. In this model, neutrophils line up along the BMZ and cause detachment of the dermal-epidermal junction through the release of proteolytic enzymes [20]. Mouse models deficient in neutrophils do not form blisters, and similar resistance is seen when neutrophil infiltration is blocked [47]. Eosinophils similarly play a critical role in the development of BP lesions, with early infiltration of the upper dermis causing release of various cytokines involved in chemotaxis, as well as tissue-damaging mediators, including matrix metalloproteinases, elastase, and gelatinase. BP patients tend to have a peripheral eosinophilia, supporting their important role in the disease [48]. Blister fluid has been found to have elevated levels of IL-5, which is known to support growth and activation of eosinophils. This fluid has also been found to have a high level of eotaxin, a chemokine responsible for the regulation of eosinophil migration [49]. It is believed that IL-5 and eotaxin heighten the inflammatory response, leading the granulocyte recruitment, which in turn leads to the release of proteinases, including eosinophil major basic protein (MBP) and eosinophilic cationic protein (ECP). These cytotoxic agents ultimately lead to the detachment of the epidermis from the dermis in the lamina lucida of the BMZ [29].

\section{Role of Proteolytic Enzymes}

Blister fluid and lesional biopsies from BP patients show high levels of proteolytic enzymes, such as NE and MMP-9, which in turn degrade both extracellular matrix proteins and the extracellular domain of BP180 [50]. Mouse models deficient in NE or MMP-9 are resistant to the disease [51, 52]. In early blister formation, MMP-9 is predominantly activated by plasmin, which is formed by the tissue plasminogen activator (tPA) and/or urokinase plasminogen activator (uPA)-mediated 
degradation of plasminogen. Stimulation with autoantibodies to BP180 has been shown to cause elevated expression and release of tPA from control keratinocytes. The mast-cell specific protease (MCP-4) (chymase) is also known to activate MMP-9. This activation in turn inactivates $\alpha 1$-proteinase inhibitor, which is responsible for the inhibition of NE. Ultimately, this leads to uninhibited activity of NE [53]. Thus, proteolytic enzyme release from inflammatory cells causes direct damage to the BMZ, leading to detachment of the dermis from the epidermis.

\section{Direct Mechanisms}

There is some evidence to suggest that autoantibodies directly interfere with the adhesion of autoantigens, in the absence of inflammatory cell action. It is hypothesized that binding of the antibody variable region to the BP180 ectodomain leads to blister formation by competing with its natural ligand. The fact that non-complement-fixing IgG4 subclass autoantibodies make up the majority of BP autoantibodies supports this theory [7]. However, research by Mihai et al. disputes this theory, showing that IgG4 is capable of leukocyte recruitment and inflammatory cell activation, despite a lack of complement involvement [54]. Additional potential direct mechanisms of blister induction may involve intracellular signaling pathways, resulting in the disassembly of hemidesmosomes and cytokine release. BP180 autoantibodies have been shown to modulate the expression of IL- 6 and IL- 8 in cultured human keratinocytes, the relevance of which is the subject of future investigation [55].

\section{Mucous Membrane Pemphigoid}

\section{Pathophysiology}

MMP is a chronic blistering disease that predominantly affects the mucous membranes, but can also affect the skin. Direct immunofluorescence studies characteristically show IgG, $\mathrm{IgA}$, or $\mathrm{C} 3$ along the $\mathrm{BMZ}$.

Seventy percent of MMP patients have BP180 as the target antigen [56]. Approximately half of MMP patients have BP180 NC16A antibodies, and the C-terminal domain is targeted [57, 58]. MMP patients have been shown to have antibodies that recognize BP230, 97/120 kDa linear IgA bullous dermatosis (LAD) antigen, laminin 332 (laminin 5) and 331 (laminin 6), and type VII collagen [59, 60]. Studies have also linked oral MMP to the $\alpha 6$-integrin subunit, and both generalized and ocular MMP to the $\beta 4$-integrin subunit [61].

An in vivo study by Lazarova et al. demonstrated that passive transfer of anti-laminin $332 \mathrm{IgG}$ to mice led to subepidermal blistering of both skin and mucous membranes in a manner consistent with MMP [62]. The clinical picture of MMP is also induced in mice injected with Fab fragments targeting laminin 332, mice lacking complement, mast cells or T cells [63]. A human model by Lazarova et al. reinforced the pathogenicity of anti-laminin 332 [64]. There is mounting evidence that the scarring process in ocular MMP may be due to pemphigoid fibroblasts, which secrete fibrogenic cytokines, matrix metalloproteinases, and collagen type I $[65,66]$. Lambiase et al. noted that conjunctival biopsies from MMP patients had significantly increased Th17 lymphocytes as compared with biopsies from healthy controls [67]. MMP lesional biopsy examinations have observed infiltrates with a high density of CD4+ T cells and Langerhans cells, which supports a role of cellular immunity in MMP $[68,69]$.

\section{Conclusion}

Our understanding of pemphigoid pathobiology has greatly expanded in recent years. Animal models have provided useful insights into mechanisms involved in the disease. Future research involving central and peripheral tolerance as well as the inflammatory mechanisms induced by autoantibody binding will be critical in developing new therapeutic agents to combat both BP and MMP.

\section{PEMPHIGUS}

\section{Introduction}

Pemphigus refers to a family of autoimmune bullous diseases characterized morphologically by widespread blisters affecting the skin and mucous membranes. PV and pemphigus foliaceus (PF), which represent the main variants of the disease, demonstrate acanotholysis caused by a loss in cell-to-cell adhesion between keratinocytes.

\section{Autoantibodies}

Autoantibodies play a critical role in the pathogenesis of PV. The desmosomal proteins, desmoglein (Dsg)-3 and Dsg1 have been shown to be target antigens in the disease. Dsg1 and Dsg3 are type 1 integral membrane glycoproteins that belong to the cadherin group of calcium-dependent cell adhesion molecules, which make up the extracellular domain of desmosomes, known as intercellular junctions, and function to maintain tissue integrity. Experimental evidence has shown that Dsg3 is expressed in the oral mucosa, particularly in the upper two-thirds, and is less strongly expressed in the epidermis, where it can be found exclusively in the basal and suprabasal layers. On the other hand, Dsg1 is expressed throughout the epidermis and oral mucosa, with overexpression occurring in the subcorneal layer of the epidermis [70]. In light of the differential expression of Dsg1 and Dsg3, 
Mahoney et al. proposed the "desmoglein compensation hypothesis", which correlates pemphigus phenotype with anti-Dsg profile and maintains that either Dsg alone is capable of maintaining the adhesion of keratinocytes. This theory rests on the following postulates: (1) in PF patients, antiDsg1 antibody alone can cause blisters in the superficial epidermis, where there is expression of Dsg1 without Dsg3; (2) Dsg3 antibodies alone cause blisters in mucosaldominant PV; and (3) mucocutaneous PV blisters occur in the presence of both Dsg1 and Dsg3 antibodies. The authors state that the mutual compensatory action of Dsg1 and Dsg3 explains the various patterns of blistering that can occur. For example, anti-Dsg3 antibodies alone do not cause cutaneous blisters due to the compensatory action of Dsg1, which is coexpressed in the skin. Conversely, in the mucosa, anti-Dsg3 antibodies alone do cause blisters, due to the inadequate expression of Dsg1 in these tissues. Similarly, the presence of both anti-Dsg3 and anti-Dsg1 antibodies leads to widespread mucocutaneous blisters [71].

In recent years, many researchers have postulated that the Dsg compensation hypothesis does not account for the whole picture of PV pathophysiology. Some take issue with the assumption that Dsg1 and 3 are entirely responsible for maintaining the integrity of the epidermis and oral mucosa. If that were indeed true, the simultaneous presence of anti-Dsg1 and anti-Dsg3 antibodies would result in epithelial disintegration into a single layer of cells, which does not occur in PV. Investigators argue that, while Dsg1 and Dsg3 are still considered the most critical autoantibodies in PV, beyond them there are complicated interactions between several known desmosmal cadherins, including Dsg2, Dsg4, and desmocollin (Dsc) 1-3. Some argue specifically that Dsg3 alone is insufficient to maintain epidermal cohesion. Research by Chen et al. highlighted this point, showing that $D s c 3^{\text {null }}$ mutant mice develop suprabasal acantholysis and clinically apparent blisters, despite the presence of Dsg3 [72].

Antibodies targeting a member of the peripheral myelin protein (PMP)-22/gas3 family, known as PERP, have been an area of recent interest. PERP-null mice display features clinically identical to PV. These findings occur despite the presence of intact Dsg3 [73]. Several non-Dsg targets have also been found to be pathogenic in PV. Nyugen et al. treated keratinocytes with antibodies to $\alpha 9$ acetylcholine receptors (AChRs), part of a novel class of cholinergic receptors with both muscarinic and nicotinic properties, and detected PV-like immunofluorescence and histology [74]. Similarly, pemphaxin (human annexin 9), which acts on cholinergic receptors, has been found to play in role in acantholysis. It has been shown that preabsorption of PV sera with pemphaxin blocks acantholysis, and permits acantholysis when added to preabsorbed PV-IgG [75]. Marchenko et al. recognized another potentially pathogenic antibody: the anti-mitochondrial antibodies. In both in vitro and in vivo studies, the authors found that absorption of these antibodies prevented the ability of PV-IgG to cause acantholysis [76]. A recent study by Tucker et al. found that expression of plakophilin-1 (PKP-1), an armadillo family protein, transforms desmosome adhesion from a calcium-dependent to a calcium-independent and hyperadhesive state, and is potentially a key regulator of desmosomal cell adhesion [77•]. The 'multiple hit' hypothesis explains the complex interplay of several antibodies in PV. This theory holds that the disruption of epidermal intergrity in PV is caused by the synergistic action of autoantibodies targeting keratinocyte cell membranes, including molecules that maintain cell shape and adhesion (AChRs), as well as molecules that control cell-cell adhesion (desmosomes). The ratio of these pathogenic autoantibodies gives rise to the range in disease severity and clinical picture across PV patients [78].While the exact interaction of these antibodies has yet to be delineated, it is generally believed that antibodies to Dsgs are the most important factors.

\section{T- and B-Cell Regulation}

Animal studies have shown that both T and B cells with Dsg3 autoreactivity are critical elements in the pathogenesis of PV. T-cell tolerance occurs through central and peripheral mechanisms. Peripherally, tolerance occurs through the inactivation of autoreactive $\mathrm{T}$ cells that have left the thymus, through $\mathrm{CD} 4+\mathrm{CD} 25+$ natural $\mathrm{T}$ regulatory cells (nTreg). These regulatory cells are unique in their expression of the transcription factor forkhead box P3 (FoxP3). Dsg3-specific type $1 \mathrm{~T}$ regulatory (Tr1) cells express FoxP3, and are found at higher frequencies in healthy carriers of PV-associated HLA class II alleles as compared with PV patients [79]. In a Dsg3 -/-mouse model, Treg cells suppressed anti-Dsg3 IgG secretion [80]. This evidence has led to the suggestion that FoxP3 modulation could restore immunologic tolerance PV. Tr1-cell activation is known to occur upon exposure to its corresponding antigen, resulting in the secretion of IL-10, an immunosuppressive cytokine. A higher proportion of Th2 to Th1 cells has been detected in PV patients, as opposed to healthy carriers of PVassociated HLA class II alleles. This finding supports the notion that Tr1 deficiencies may be due to a loss of Dsg3 tolerance in PV, in accordance with low peripheral Treg cell levels in active PV [81].

Several cytokines have been linked to PV pathogenesis, including IL-4, IL-5, Il-6, and IL-10, which suggest Th2 involvement. Th1 cells also appear to be necessary for PV to occur [81]. Th1 cells have predominantly been noted in patients with chronic active PV, as well as healthy carriers of PV-associated HLA class II alleles; however, healthy patients seem to lack Dsg3-reactive Th2 cells. Both Th1 and Th2 cells are apparent in the acute phase of PV, suggesting that both Th subtypes are critical in various phases of the disease course [82]. 
Depletion of CD4+T cells has been shown to decrease serum levels of anti-Dsg3 antibodies in PV patients, although the complexities of T-cell regulation of B-cell function are still under investigation [83]. Strong evidence has shown that B cells are activated by Dsg3-specific autoreactive T cells through IL-4 secretion [84]. CD40L (CD154) binding on activated T cells with CD40 on B cells is considered critical to the interaction of activated $\mathrm{T}$ and $\mathrm{B}$ cells. It has also been shown that anti-CD154 mAb is capable of blocking this interaction between CD40 and CD154, resulting in the suppression of anti-Dsg3 IgG production [85]. Future investigations into autoreactive T-cell regulators of B-cell antibody production are needed to shed light on more targeted therapeutic interventions.

\section{Mechanisms Leading to Acantholysis}

Pemphigus research has elucidated several mechanisms for keratinocyte detachment. Phosphorylation of several target keratinocyte proteins and activation of signaling events play a role in the complex process leading to acantholysis and cell death. E-cadherin, Dsg3, and $\beta-, \gamma-$, and p120 catenins are known substrates of PV-IgG-dependent phosphorylation [86, 87]. Aoyama and Kitajima showed that the binding of PV-IgG to cell surface targets induces Dsg3 phosphorylation and leads to its separation from plakoglobin, as well causes rapid depletion of Dsg3 from desmosomes [88].

Several studies have shown that desmosomal separation occurs in the setting of widened intercellular spaces. This separation is caused by the tension of shearing forces, which rips intact desmosomes from the cell membrane. The 'basal cell shrinkage' theory explains why acantholysis in PV tends to occur in the basal layer, leading to the characteristic 'tombstoning' appearance. This theory holds the following: (1) epidermal cells dissociate due to shrinkage, which overpowers the forces exerted by the desmosome; (2) basal cells shrink more than suprabasal cells, leading to suprabasal clefting; (3) complex signal transduction pathways initiate cytoskeleton rupture and keratinocyte shrinkage [89].

In recent years, some of the complex molecular mechanisms involved in PV have been identified. A recent mouse model showed that neonatal mice injected with PV-IgG did not develop acantholysis when pretreated with the mammalian target of rapamycin (mTOR) inhibitor, sirolimus. Furthermore, the upregulation of mTOR noted in basal keratinocytes as well as signs of apoptosis were abolished in the presence of sirolimus [90]. PV antibody-mediated keratinocyte apoptosis has been shown to follow the cMyc-dependent pathway, which involves caspase 9 [91]. It is known that cyclin-dependent kinase 2 (Cdk2) is necessary for c-Myc-mediated apoptosis. In a PV mouse model, Lanza et al. found that injection with PV sera lead to overexpression of Cdk2 [92]. These results support the consistent finding of selective acantholysis of the basal cell layer, despite the fact that $\operatorname{IgG}$ antibodies deposit throughout the epidermis. Several other antibodies, including anti-PERP PV and anti-mitochochondrial antibodies, have been shown to initiate apoptotic pathways in epidermal cells $[76,93]$. The Fas ligand (FasL) has been shown to trigger apoptosis in PV sera through the activation of caspase-8 [94]. Giurdanella et al. examined the passive transfer of PV autoantibodies using CD8-deficient mice, and found that these mice showed a lower incidence of PV. They also used immunohistochemical staining to show that $\mathrm{CD} 4+\mathrm{T}$ cells are more abundantly expressed than CD8+ T cells in the inflammatory infiltrate of PV lesions. The authors hypothesized that CD8+ T cells act through the Fas/FasL pathway [95•]. Serum TNF $\alpha$ has also been shown to correlate with disease activity, while antiTNF $\alpha$ antibodies have been shown to inhibit PV acantholysis in vitro $[96,97]$.

The term 'apoptolysis' has been proposed to connect acantholysis with cell death pathways. These processes share the same set of cell death enzymes, as well signal effectors as triggers due to PV-IgG binding. Grando proposed that apoptolysis occurs through the following events: (1) antibodies bind keratinocyte receptor ligands, sending a series of agonist- and antagonist-like signals; (2) the cell death enzymatic cascade is triggered by the combined activation of Src, epidermal growth factor receptor kinase (EGFRK), p38 mitogen-activated protein kinase (MAPK) and mTOR, as well as downstream signaling elements and elevated intracellular $\mathrm{Ca}^{2+}$; (3) the reorganization of cortical actin filaments and collapse of tonofilaments cleaved by apoptotic caspases, as well as the separation of intercellular adhesion complexes due to adhesion molecule phosphorylation and caspase cleavage initiates basal cell shrinkage leading to suprabasal acantholysis; (4) acantholysis escalates when apoptotic enzymes cause the widespread collapse of cytoskeletal proteins, leading to shearing forces on desmosomes and separation of collapsing cells, which activates the production of scavenging antibodies; (5) damaged mitochondrial and nuclear proteins precede the recruitment and death of acantholytic cells in the lower epidermis [78]. This hypothesis is critical in that it connects these two essential components in the pathophysiology of PV. Further research is needed to delineate the initiating event - apoptosis or acantholysis. A recent study by Mao et al. expanded on some of these mechanisms of blister formation through the identification of MAPK-activated protein kinase 2 (MK2) as a key downstream effector of p38 cell signaling in the passive transfer of human anti-Dsg IgG4 mAbs to neonatal mice [98•].

\section{Conclusions}

While much has been learned in recent years, the pathogenesis of PV remains controversial. Several animal models have 
expanded our understanding of the mechanisms behind the disease. Clearly, Dsg autoantibodies play the most critical role in the disease, with various other target antigens acting adjunctively to exacerbate the disease by apoptotic signaling. The relationship between apoptosis and acanthosis has yet to be clearly defined, but they may promote each other to enhance disease activity. Both $\mathrm{T}$ and $\mathrm{B}$ cells with Dsg3 autoreactivity are critical in the pathogenesis of PV. Future research is needed to further investigate autoreactive T-cell regulators of B-cell antibody production. Future investigation into many novel molecular and antigenic targets will help to more clearly elucidate our understanding of PV disease pathogenesis.

Acknowledgments This material is based upon work supported by the Department of Veterans Affairs (Veterans Health Administration, Office of Research and Development, Biomedical Laboratory Research and Development) to Victoria Werth.

\section{Compliance with Ethics Guidelines}

Conflict of Interest Dr. Lauren Okon declares no potential conflicts of interest. Dr. Victoria Werth is Head of the Medical Advisory Board, International Pemphigus and Pemphigoid Foundation (IPPF).

Human and Animal Rights and Informed Consent This article does not contain any studies with human or animal subjects performed by any of the authors.

\section{References}

Papers of particular interest, published recently, have been highlighted as:

- Of importance

1. Ujiie H, Nishie W, Shimizu H. Pathogenesis of bullous pemphigoid. Dermatol Clin. 2011;29:439-46.

2. Diaz LA, Ratrie 3rd H, Saunder WS, et al. Isolation of a human epidermal cDNA corresponding to the $180-\mathrm{kD}$ autoantigen recognized by bullous pemphigoid and herpes gestationis sera. Immunolocalization of this protein to the hemidesmosome. J Clin Invest. 1990;86:1088-94.

3. Li K, Sawamura D, Giudice GJ, Diaz LA, Mattei MG, Chu ML, et al. Genomic organization of collagenous domains and chromosomal assignment of human $180-\mathrm{kD}$ bullous pemphigoid antigen-2, a novel collagen of stratified squamous epithelium. J Biol Chem. 1991;266:24064-9.

4. Sawamura D, Nomura K, Sugita Y, Mattei M, Chu M, Knowlton R, et al. Bullous pemphigoid antigen (BPAG1): cDNA cloning and mapping of the gene to the short arm of human chromosome 6 . Genomics. 1990;8:722-6.

5. Stanley JR, Tanaka T, Mueller S, Klaus-Kovtun V, Roop D. Isolation of complementary DNA for bullous pemphigoid antigen by use of patients' autoantibodies. J Clin Invest. 1988;82:1864-70.

6. Giudice GJ, Emery DJ, Diaz LA. Cloning and primary structural analysis of the bullous pemphigoid autoantigen BP180. J Invest Dermatol. 1992;99:243-50.
7. Schiavo AL, Ruocco E, Brancaccio G, Caccavale S, Ruocco V, Wolf R. Bullous pemphigoid: etiology, pathogenesis, and inducing factors: facts and controversies. Clin Dermatol. 2013;31:391-9.

8. Giudice GJ, Emery DJ, Zelickson BD, Anhalt GJ, Liu Z, Diaz LA. Bullous pemphigoid and herpes gestationis autoantibodies recognize a common non-collagenous site on the BP180 ectodomain. J Immunol. 1993;151:5742-50.

9. Leyendeckers H, Tasanen K, Bruckner-Tudermann L, Zillikens D, Sitaru C, Schmitz J, et al. Memory B cells specific for the NC16A domain of the $180 \mathrm{kDa}$ bullous pemphigoid autoantigen can be detected in the peripheral blood of bullous pemphigoid patients and induced in vitro to synthesize autoantibodies. J Invest Dermatol. 2003; 120:372-8.

10. Hofmann S, Thoma-Uszynski S, Hunziker T, et al. Severity and phenotype of bullous pemphigoid relate to autoantibody profile against the NH2- and $\mathrm{COOH}$-terminal regions of the BP180 ectodomain. J Invest Dermatol. 2002;119:1065-73.

11. Schmidt E, Obe K, Brocker EB, Zillikens D. Serum levels of autoantibodies to BP180 correlate with disease activity in patients with bullous pemphigoid. Arch Dermatol. 2000;136:174-8.

12. Perriard J, Jaunin F, Favre B, et al. IgG autoantibodies from bullous pemphigoid (BP) patients bind antigenic sites on both the extracellular and intracellular domains of the BP antigen 180. J Invest Dermatol. 1999;112:141-7.

13. Di Zenzo G, Grosso F, Terracina M, et al. Characterization of the anti-BP180 autoantibody reactivity profile and epitope mapping in bullous pemphigoid patients. J Invest Dermatol. 2004;122:103-10.

14. Kromminga A, Scheckenbach C, Georgi M, et al. Patients with bullous pemphigoid and linear IgA disease show a dual IgA and IgG autoimmune response to BP180. J Autoimmun. 2000;15:293300.

15. Dopp R, Schmitd E, Chimanovitch I, Leverkus M, Brocker EB, Zillikens D. IgG4 and IgE are the major immunoglobulins targeting the NC16A domain of BP180 in bullous pemphigoid: serum levels of these immunoglobulins reflect disease activity. J Am Acad Dermatol. 2000;42:577-83.

16. Christophoridis S, Budinger L, Borradori L, Hunziker T, Merk HF, Hertl M. IgG, $\operatorname{IgA}$, and $\operatorname{IgE}$ autoantibodies against ectodomain of BP180 in patients with bullous and cicatricial pemphigoid and linear IgA bullous dermatosis. Br J Dermatol. 2000;143:349-55.

17. Skaria M, Jaunin F, Hunziker T, et al. IgG autoantibodies from bullous pemphigoid patients recognize multiple antigenic reactive sites located predominantly within the $\mathrm{B}$ and $\mathrm{C}$ subdomains of the COOH-terminus of BP230. J Invest Dermatol. 2000;114:998-1004.

18. Di Zenzo G, Thoma-Uszynski S, Calabresi V, et al. Demonstration of epitope-spreading phenomena in bullous pemphigoid: results of a prospective multicenter study. J Invest Dermatol. 2011;131: 2271-80.

19. Gammon WR, Merritt CC, Lewis DM, Sams Jr WM, Carlo JR, Wheeler Jr CE. An in vitro model of immune complex-mediated basement membrane zone separation caused by pemphigoid antibodies, leukocytes, and complement. J Invest Dermatol. 1982;78: 285-90.

20. Sitaru C, Schmidt E, Petermann S, Munteanu LS, Brocker EB, Zillikens D. Autoantibodies to bullous pemphigoid antigen 180 induce dermal-epidermal separation in cryosections of human skin. J Invest Dermatol. 2002;118:664-71.

21. Bieber K, Sun S, Ishii N, et al. Animal models for autoimmune bullous dermatoses. Exp Dermatol. 2009;19:2-11.

22. Liu Z, Diaz LA, Troy JL, Taylor AF, Emeery DE, Fairley JA, et al. A passive transfer model of the organ-specific autoimmune disease, bullous pemphigoid, using antibodies generated against the hemidesmosomal antigen, BP180. J Clin Invest. 1993;92:2480-8.

23. Lin L, Bankaitis E, Heimbach L, et al. Dual targets for mouse mast cell protease-4 in mediating tissue damage in experimental bullous pemphigoid. J Biol Chem. 2011;286:37358-67. 
24. Fairley JA, Burnett CT, Fu CL, Larson DL, Fleming MG, Giudice GJ. A pathogenic role for IgE in autoimmunity: bullous pemphigoid IgE reproduces the early phase of lesion development in human skin grafter to nu/nu mice. J Invest Dermatol. 2007;127: 2605-11.

25. Zone JJ, Taylor T, Hull C, Schmidt L, Meyer L. IgE basement membrane zone antibodies induce eosinophil infiltration and histological blisters in engrated human skin on SCID mice. J Invest Dermatol. 2007;127:1167-74.

26. Messingham KN, Srikantha R, DeGueme AM, Fairley JA. FcRindependent effects of $\operatorname{IgE}$ and $\operatorname{IgG}$ autoantibodies in bullous pemphigoid. J Immunol. 2011;187(1):553-60.

27. Fairley JA, Baum CL, Brandt DS, Messingham KA. Pathogenicity of IgE in autoimmunity: successful treatment of bullous pemphigoid with omalizumab. J Allergy Clin Immunol. 2009;123:704-5.

28. Hall RPI, Murray JC, McCord MM, Rico JM, Streilein RD. Rabbits immunized with a peptide encoded by the $230-\mathrm{kD}$ bullous pemphigoid antigen cDNA develop an enhanced inflammatory response to UVB irradiation: a potential animal model for bullous pemphigoid. J Invest Dermatol. 1993;101:9-14.

29. Kasperkiewicz M, Zillikens D. The pathophysiology of bullous pemphigoid. Clin Rev Allerg Immunol. 2007;33:67-77.

30. Kiss M, Husz S, Janossy T, et al. Experimental bullous pemphigoid generated in mice with an antigenic epitope of the human hemidesmosomal protein BP230. J Autoimmun. 2005;24:1-10.

31. Rensing-Ehl A, Gaus B, Bruckner-Tuderman L, Martin SF. Frequency, function and CLA expression of CD4+CD25+ FOXP3+ regulatory $\mathrm{T}$ cells in bullous pemphigoid. Exp Dermatol. 2007;16(1):13-21.

32. Schlomchik MJ. Activating systemic autoimmunity: B's, T's, and tolls. Curr Opin Immunol. 2009;21:626-33.

33. D'Auria L, Cordiali Fei P, Ameglio F. Cytokines and bullous pemphigoid. Eur Cytokine Netw. 1999;10:123-34.

34. Li Q, Liu Z, Dang E, Jin L, He Z, Yang L, et al. Follicular helper T cells (Tfh) and IL-21 involvement in the pathogenesis of bullous pemphigoid. PLoS One. 2013;8(7):e68145. In this study, the authors investigated the frequencies of Tfh in the peripheral blood of $B P$ patients. They found a significantly higher Tfh cell count in $B P$ patients as compared with healthy controls $(p<0.001)$. They also noted higher serum IL-21 levels in BP patients as compared with healthy controls $(p<0.001)$. They found that the frequencies of both Tfh cells and IL-21 were both positively correlated with antiBP180-NC16A autoantibody titers $(R=0.712, p<0.01$; and $R=$ $0.578, p=0.030$, respectively). They also noted that both of these levels decreased as the patients improved clinically after treatment. Finally, they noted that Tfh depleted CD4+T cells, and anti-IL-21 neutralization antibody could block the T-cell-induced B-cell activation and secretion of BP autoantibody in vitro.

35. Toosi S, Bystryn JC. Potential role of interleukin-17 in the pathogenesis of bullous pemphigoid. Med Hypothesis. 2010;74:727-8.

36. Arakawa M, Dainichi T, Tshii N, et al. Lesional Th17 cells and regulatory T cells in bullous pemphigoid. Exp Dermatol. 2011;20: 1011-37.

37. Zebrowska A, Wagrowska-Danilewicz M, Danilewicz M, Stasikowska-Kanicka O, Cynkier A, Sysa-Jedrzejowska A, et al. IL-17 expression in dermatitis herpetiformis and bullous pemphigoid. Mediators Inflamm. 2013;967987. This study assessed IL-17 expression in biopsies from patients with dermatitis herpetiformis (DH) and BP. The authors evaluated $10 \mathrm{DH}$ patients, $14 \mathrm{BP}$ patients, and 10 healthy controls through immunohistochemical staining of biopsies and serum concentration as measured by immunoassays. Results showed IL-17 expression was statistically higher in the epidermis of BP specimens as compared with $\mathrm{DH}$ specimens, and was not observed in biopsies from healthy controls. They also found that serum level of IL-17 was statistically higher in both BP and DH patients as compared with controls. The results provide evidence for a potentially important role of IL-17 in these disease processes.

38. Hertl M, Eming R, Veldman C. T cell control in autoimmune bullous skin disorders. J Clin Invest. 2006;116:1159-66.

39. Provost TT, Tomasi Jr TB. Evidence for complement activation via the alternative pathway in skin diseases. Herpes gestationis, systemic lupus erythematosus, and bullous pemphigoid. J Clin Invest. 1973;52:1779-87.

40. Suzuki M, Harada S, Yaoita Y. Purification of bullous pemphigoid IgG subclasses and their capability for complement fixation. Acta Derm Venerol. 1992;72:245-9.

41. Jordan RE, Kawana S, Fritz KA. Immunopathologic mechanisms in pemphigus and pemphigoid. J Invest Dermatol. 1985;85:72-8.

42. Liu Z, Giudice GJ, Swartz SJ, Fairley JA, Till GO, Troy JL, et al. The role of complement in experimental bullous pemphigoid. J Clin Invest. 1995;4:1539-44.

43. Wintroub BU, Mihm Jr MC, Goetzl EJ, Soter NA, Austen KF. Morphologic and functional evidence for release of mast-cell products in bullous pemphigoid. N Engl J Med. 1978;298:417-21.

44. Chen R, Ning G, Zhao ML, Fleming MG, Diaz LA, Werb Z, et al. Mast cells play a key role in neutrophil recruitment in experimental bullous pemphigoid. J Clin Invest. 2001;108:1151-8.

45. Dimson OG, Giudice GJ, Fu CL, Van Den Bergh F, Warren SJ, Janson MM, et al. Identification of a potential effector function for $\operatorname{IgE}$ autoantibodies in the organ-specific autoimmune disease bullous pemphigoid. J Invest Dermatol. 2003;120:784-8.

46. Iryo K, Tsuda S, Sasai Y. Ultrastructural aspects of infiltrated eosinophils in bullous pemphigoid. J Dermatol. 1992;19:393-9.

47. Liu Z, Giudice GJ, Zhou X, Swartz SJ, Troy JL, Fairley JA, et al. A major role for neutrophils in experimental bullous pemphigoid. $\mathrm{J}$ Clin Invest. 1997;100(5):1256-63.

48. Bushkell LL, Jordan RE. Bullous pemphigoid: a cause of peripheral blood eosinophilia. J Am Acad Dermatol. 1983;8:648-51.

49. Wakugawa M, Nakamura K, Hino H, Toyama K, Hattori N, Okochi $\mathrm{H}$, et al. Elevated levels of eotaxin and interleukin-5 in blister fluid of bullous pemphigoid: correlation with tissue eosinophila. Br J Dermatol. 2000;143:112-6.

50. Ståhle-Bäckdahl M, Inoue M, Guidice GJ, Parks WC. 92-kD gelatinase is produced by eosinophils at the site of blister formation in bullous pemphigoid and cleaves the extracellular domain of recombinant $180-\mathrm{kD}$ bullous pemphigoid autoantigen. J Clin Invest. 1994;93:2022-30.

51. Liu Z, Shipley JM, Vu TH, Zhou X, Diaz LA, Werb Z, et al. Gelatinase B-deficient mice are resistant to experimental BP. J Exp Med. 1998;188:475-82.

52. Liu Z, Shapiro SD, Zhou X, Twining SS, Senior RM, Giudice GJ, et al. A critical role for neutrophil elastase in experimental bullous pemphigoid. J Clin Invest. 2000;105:113-23.

53. Liu Z, Zhou X, Shapiro SD, Shipley JM, Twining SS, Diaz LA, et al. The serpin alpha1-proteinase inhibitor is a critical substrate for gelatinase B/MMP-9 in vivo. Cell. 2000;102:647-55.

54. Mihai S, Chiriac MT, Herrero-González JE, Goodall M, Jefferis R, Savage CO. IgG4 autoantibodies induce dermal-epidermal separation. J Invest Dermatol. 2000;115:842-8.

55. Schmidt E, Reimer S, Kruse N, Jainta S, Bröcker EB, Marinkovich MP, et al. Autoantibodies to BP180 associated with bullous pemphigoid release interleukin- 6 and interleukin- 8 from cultured human keratinocytes. J Invest Dermatol. 2000;115(5):842-8.

56. Kasperkiewicz M, Zillikens D, Schmidt E. Pemphigoid diseases: pathogenesis, diagnosis, and treatment. Autoimmunity. 2012;45(1): 55-70.

57. Schmidt E, Skrobek C, Kormminga A, Hashimoto T, Messer G, Brocker EB, et al. Cicatracial pemphigoid: IgA and IgG autoantibodies target epitopes on both intra- and extracellular domains of bullous pemphigoid antigen 180. Br J Dermatol. 2001;145: $778-83$. 
58. Calabresi V, Carrozza M, Cozzani E, Arduino P, Bertolusso G, Tirone F, et al. Oral pemphigoid autoantibodies preferentially target BP180 ectodomain. Clin Immunol. 2007;122:207-13.

59. Domlage-Hultsch N, Gammon WR, Briggaman RA, Gil SG, Carter WG, Yancey KB. Epiligrin, the major human keratinocyte intergrin ligand, is a target in both an acquired autoimmune and an inherited subepidermal blistering skin disease. J Clin Invest. 1992;90:1628-33.

60. Chan LS, Majmudar AA, Tran HH, Meier F, Schaumburg-Lever G, Chen M, et al. Laminin- 6 and laminin- 5 are recognized by autoantibodies in a subset of cicatracial pemphigoid. J Invest Dermatol. 1997;108:848-53.

61. Rashid KA, Gurcan HM, Ahmed AR. Antigen specificity in subsets of mucous membrane pemphigoid. J Invest Dermatol. 2006;126: 2631-6.

62. Lazarova Z, Yee C, Darling T, Briggaman RA, Yancey KB. Passive transfer of anti-laminin 5 antibodies induces subepidermal blisters in neonatal mice. J Clin Invest. 1996;98:1509-18.

63. Lazarova Z, Hsu R, Briggaman RA, Yancey KB. Fab fragments directed against laminin 5 induce subepidermal blisters in neonatal mice. Clin Immunol. 2000;95:26-32.

64. Lazarova Z, Hsu R, Yee C, Yancey KB. Human anti-laminin 5 autoantibodies induce subepidermal blisters in an experimental human skin graft model. J Invest Dermatol. 2000;114:178-84.

65. Razzaque MS, Foster CS, Amhed AR. Tissue and molecular events in human conjunctival scarring in ocular cicatracial pemphigoid. Histol Histopathol. 2001;16:1203-12.

66. Saw VP, Schmidt E, Offiah I, Galatowicz G, Zillikens D, Dart JK, et al. Profibrotic phenotype of conjunctival fibroblasts from mucous membrane pemphigoid. Am J Pathol. 2011;178:187-97.

67. Lambiase A, Micera A, Mantelli F, et al. T-helper 17 lymphocytes in ocular cicatricial pemphigoid. Mol Vis. 2009;15:1449-55.

68. Bernauer W, Wright P, Dart JK, et al. The conjunctiva in acute and chronic mucous membrane pemphigoid: an immunohistochemical analysis. Opthalmology. 1993;100:339-46.

69. Bodaghi B, Bertin V, Paques M, et al. Limbal conjunctival Langerhans cell density in ocular cicatricial pemphigoid: an indirect immunofluourescence studyon Dispase-split conjunctiva. Curr Eye Res. 1997;16:820-4.

70. Cirillo N, Cozzani E, Grando SA. Urban legends: pemphigus vulgaris. Oral Dis. 2012;18:442-58.

71. Mahoney MG, Wang Z, Rothernberger K, Koch PJ, Amagai M, Stanley JR. Explanations for the clinical and microscopic localization of lesions in pemphigus foliaceus and vulgaris. J Clin Invest. 1999;103:461-8

72. Chen J, Den Z, Koch PJ. Loss of desmocollin 3 in mice leads to epidermal blistering. J Cell Sci. 2008;121:2844-9.

73. Irhie RA, Marques MR, Nguyen BT, Horner JS, Papazoglu C, Bronson RT, et al. Perp is a p63-regulated gene essential for epithelial integrity. Cell. 2005;120:843-56.

74. Nguyen VT, Ndoye A, Grando SA. Novel human alpha9 acetylcholine receptor regulating keratinocyte adhesion is targeted by pemphigus vulgaris autoimmunity. Am J Pathol. 2000;157: 1377-91.

75. Nguyen VT, Ndoye A, Grando SA. Pemphigus vulgaris antibody identifies pemphaxin, a novel keratinocyte annexin-like molecule binding acetylcholine. J Biol Chem. 2000;275:29466-76.

76. Marchenko S, Chernyavsky AI, Arredondo J, Gindi V, Grando SA. Antimitochondrial antibodies in pemphigus vulgaris: a missing link in disease pathophysiology. J Biol Chem. 2010;285:3695-704.

77. Tucker DK, Stahley SN, Kowalczyk AP. Plakophilin-1 protects keratinocytes from pemphigus vulgaris IgG by forming calciumdependent desmosomes. J Invest Dermatol. 2014;134:1033-43. Tucker et al. studied a series of Dsg3 chimeras and deletion constructs and noted that PKP-1, an armadillo family protein, clusters Dsg3 with the desmosomal plaque protein desmoplakin in a way that is dependent upon the plakoglobin-binding domain of the Dsg3 tail. They also noted that PKP-1 expression alters desmosome adhesion from a calcium-dependent to a calcium-independent and hyperadhesive state. The authors commented that manipulation of this single desmosomal plaque protein inhibits the pathogenic effects of PV-IgG on keratinocyte adhesion.

78. Grando SA. Pemphigus autoimmunity: hypotheses and realities. Autoimmunity. 2012;45(1):7-35.

79. Veldman C, Höhne A, Dieckmann D, Schuler G, Hertl M. Type I regulatory $\mathrm{T}$ cells specific for desmoglein 3 are more frequently detected in healthy individuals than in patients with pemphigus vulgaris. J Immunol. 2012;189:3778.

80. Yokoyama T, Matsuda S, Takae Y, Wada N, Nishikawa T, Amagai $\mathrm{M}$, et al. Antigen-independent development of FoxP3+ regulatory $\mathrm{T}$ cells suppressing autoantibody production in experimental pemphigus vulgaris. Int Immunol. 2011;23:365-73.

81. Amber KT, Staropoli P, Shiman MI, Elgart GW, Hertl M. Autoreactive $\mathrm{T}$ cells in the immune pathogenesis of pemphigus vulgaris. Exp Dermatol. 2013;22:699-704.

82. Hertl M, Veldman C. T-cellular autoimmunity against desmogleins in pemphigus, an autoantibody-mediated bullous disorder of the skin. Autoimmun Rev. 2003;2:278-83.

83. Nishifuji K, Amagai M, Kuwana M, Iwasaki T, Nishikawa T, et al. Detection of antigen-specific B cells in patients with pemphigus vulgaris by enzyme-linked immunospot assay: requirement of $\mathrm{T}$ cell collaboration for autoantibody production. J Invest Dermatol. 2000;114:88-94.

84. Takahashi H, Amagai M, Nishikawa T, Fujii Y, Kawakami Y, Kuwana M, et al. Novel system evaluating in vivo pathogenicity of desmoglein 3-reactive $\mathrm{T}$ cell clones using murine pemphigus vulgaris. J Immunol. 2008;181:1526-35.

85. Aoki-Ota M, Kinoshita M, Ota T, Tsunoda K, Iwasaki T, Tanaka S, et al. Tolerance induction by the blockade of Cd40/CD154 interaction in pemphigus vulgaris mouse model. J Invest Dermatol. 2006;126:105-13.

86. Nguyen VT, Arredondo J, Chernyavsky AI, Kitajima Y, Pittelkow M, Grando SA. Pemphigus vulgaris IgG and methylprednisolone exhibit reciprocal effects on keratinocytes. J Biol Chem. 2004;279: 2135-46.

87. Chernyavsky AI, Arredondo J, Piser T, Karlsonn E, Grando SA Differential coupling of M1 muscarinic and alpha7 nicotinic receptors to inhibition of pemphigus acantholysis. J Biol Chem. 2008;283:3401-8

88. Aoyama Y, Kitajima Y. Pemphigus vulgaris-IgG causes a rapid depletion of desmoglein 3 (Dsg3) from the triton X-100 soluble pools, leading to the formation of Dsg3-depleted desmosomes in a human squamous carcinoma cell line, DJM-1 cells. J Invest Dermatol. 1999;112:67-71.

89. Bystryn J-C, Grando SA. A novel explanation for acantholysis in pemphigus vulgaris: the "basal cell shrinkage" hypothesis. J Am Acad Dermatol. 2006;54:513-6.

90. Pretel M, España A, Marquina M, Pelacho B, Lopez-Picazo J, Lopez-Zabalza M. An imbalance in Akt/mTOR is involved in the apoptotic and acantholytic processes in a mouse model of pemphigus vulgaris. Exp Dermatol. 2009;18:771-80.

91. Baroni A, Buommino E, Paoletti I, Orlando M, Ruocco E, Ruocco V. Pemphigus serum and captopril induce heat shock protein 70 and inducible nitric oxide sythase overexpression, triggering apoptosis in human keratinocytes. Br J Dermatol. 2004;150:1070-80.

92. Lanza A, Cirillo N, Rossiello R, Rienzo M, Cutillo L, Casamassimi A, et al. Evidence of key role of Cdk2 overexpression in pemphigus vulgaris. J Biol Chem. 2008;283:8736-45.

93. Davies L, Gray D, Spiller D, White MR, Damato B, Grierson I, et al. P53 apoptosis mediator PERP: localization, function and caspase activation in uveal melanoma. J Cell Mol Med. 2009;13: 1995-2007. 
94. Puviani M, Marconi A, Cozzani E, Pincelli C. Fas ligand in pemphigus sera induces keratinocyte apoptosis through the activation of caspase-8. J Invest Dermatol. 2003;120:164-7.

95. Giurdanella F, Fania L, Gnarra M, Toto P, Di Rollo D, Sauder DN, et al. A possible role of CD8+ T lymphocytes in the cell-mediated pathogenesis of pemphigus vulgaris. Mediators Inflamm. 2013;764290. The authors of this study examined the role of $C D 8+T$ cells in the process of acantholysis through passive transfer of PV autoantibodies in CD8-deficient mice, as well as the inflammatory infiltrates in immunohistochemically-stained $P V$ lesional biopsies. Staining studies shown that CD4+ is expressed more than $C D 8+$ in the inflammatory infiltrate of PV lesions. They also found a lower incidence of pemphigus in CD8-deficient mice as compared with healthy controls. These results suggest that $C D 8+T$ cells may be an important element in the pathogenesis of $P V$, and the authors propose that these cells act through the Fas/ FasL pathway.

96. D’Auria L, Bonifati C, Mussi A, D’Agosto G, De Simone C, Giacalone B, et al. Cytokines in the sera of patients with pemphigus vulgaris: interleukin-6 and tumour necrosis factor-alpha levels are significantly increased as compared to healthy subjects and correlate with disease activity. Eur Cytokine Netw. 1997;8:383-7.
97. Feliciani C, Toto P, Amerio P, Pour SM, Coscione G, Shivji G, et al. In vitro and in vivo expression of interleukin-1alpha and tumour necrosis factor-alpha mRNA in pemphigus vulgaris: interleukin1 alpha and tumour necrosis factor-alpha are involved in acantholysis. J Invest Dermatol. 2000;114:71-7.

98. Mao X, Li H, Sano Y, Gaestel M, Park JM, Payne AS. MAPKAP kinase 2 (MK2)-dependent and -independent models of blister formation in pemphigus vulgaris. J Invest Dermatol. 2014;134: 68-76. The authors of this study assessed MK2 as a downstream effector of p38 signaling in $P V$, as well as elucidate both $M K$ dependent and-independent mechanisms of blister development through passive transfer of human anti-Dsg IgG4 mAbs to neonatal mice. The authors found the following: (1) in human keratinocytes, MK2 is activated by PV mAbs in a dose-dependent fashion; (2) MK2 activation occurs in human blisters, which leads to the translocation of MK2 from the nucleus to the cytosol; (3) in human keratinocytes, small-molecule inhibition of MK2 and blocking MK2 expression inhibits PV mAb-induced Dsg3 endocytosis; (4) genetic deletion of $p 38 \alpha$ and MK2 block spontaneous suprabasal blisters by PV mAbs in mouse models. These data highlight the role of MK2 as a key downstream effector of p38, and suggests the potential of MK2 for modulating PVautoantibody pathogenicity. 